OPEN ACCESS

Edited by:

Sheng S. Zhang,

United States Army Research

Laboratory, United States

Reviewed by:

Dongshuang Wu,

Kyoto University, Japan

Fudong Han,

Rensselaer Polytechnic Institute,

United States

${ }^{*}$ Correspondence:

Zhicheng Zhang

zczhang19@tju.edu.cn

Specialty section:

This article was submitted to Electrochemical Energy Conversion and Storage,

a section of the journal

Frontiers in Energy Research

Received: 06 March 2021 Accepted: 12 April 2021

Published: 10 May 2021

Citation:

Li J, Chen Y, Fan Z and Zhang Z

(2021) Editorial: Emerging Technologies for Materials Design and Characterization in Energy Conversion and Storage.

Front. Energy Res. 9:676876. doi: 10.3389/fenrg.2021.676876

\section{Editorial: Emerging Technologies for Materials Design and Characterization in Energy Conversion and Storage}

\author{
Junjun $\mathrm{Li}^{1}$, Yanan Chen ${ }^{2}$, Zhanxi Fan ${ }^{3,4}$ and Zhicheng Zhang ${ }^{1 *}$ \\ ${ }^{1}$ Tianjin Key Laboratory of Molecular Optoelectronic Sciences, Collaborative Innovation Center of Chemical Science and \\ Engineering, Department of Chemistry, School of Science, Tianjin University, Tianjin, China, ${ }^{2}$ Tianjin Key Laboratory of \\ Composite and Functional Materials, School of Materials Science and Engineering Key Laboratory of Advanced Ceramics \\ and Machining Technology of Ministry of Education, Tianjin University, Tianjin, China, ${ }^{3}$ Department of Chemistry, City \\ University of Hong Kong, Kowloon, Hong Kong, ${ }^{4}$ Hong Kong Branch of National Precious Metals Material Engineering \\ Research Center (NPMM), City University of Hong Kong, Kowloon, Hong Kong
}

Keywords: energy conversion and storage, electrocatalysis, water splitting, $\mathrm{CO}_{2}$ reduction, nitrogen reduction reaction, fuel cell

\section{Editorial on the Research Topic}

\section{Emerging Technologies for Materials Design and Characterization in Energy Conversion and Storage}

The increasing consumption of fossil fuels leads to energy crisis and environmental issues, which seriously affects human daily life. To date, great efforts have been made to explore sustainable, ecofriendly and renewable energy alternatives to fossil fuels. In the past few decades, various energy conversion and storage technologies, such as water splitting (Zhang F. et al., 2019; Hu et al., 2021; Wu et al., 2021), proton exchange membrane fuel cells (Edwards et al., 2008; Park et al., 2012), nitrogen reduction reaction (NRR) (Wan et al., 2019; Zhang W. et al., 2019; Yang et al., 2020b; Li et al., 2021), $\mathrm{CO}_{2}$ reduction reaction $\left(\mathrm{CO}_{2} \mathrm{RR}\right)$ (Ozdemir et al., 2019; Liu et al., 2020; Yang et al., 2020a; Ma et al., 2021; Wang et al., 2021), and metal-air batteries (Cheng and Chen, 2012) have shown promising potential due to the high efficiency, energy security, and environmental protection. In these fields, more attention has been paid to preparing advanced materials with outstanding performance, and developing advanced technologies for prediction, characterization and detection (Centi, 2020).

Electrocatalytic NRR to $\mathrm{NH}_{3}$ has been regarded as an attractive alternative to the traditional Haber-Bosch process owing to its lower energy consumption under ambient conditions (Tang and Qiao, 2019; Yang et al., 2020b). The development of advanced NRR catalysts with outstanding performance and low costs is highly desired. Recently, Wang et al. reported that the ringlike $\mathrm{V}_{2} \mathrm{O}_{3}$ nanostructures could effectively convert $\mathrm{N}_{2}$ to $\mathrm{NH}_{3}$ under ambient conditions. Scanning electron microscopy analysis shows that the ringlike structure is uniform with outer diameter of 350-500 nm. Transmission electron microscopy (TEM) analysis confirms that such nanoring possesses a rough surface, displaying more active sites. The high-resolution TEM image of an individual nanoring indicates a contracted interplanar distance of $0.211 \mathrm{~nm}$, corresponding to the (113) plane. This work presents a facile strategy to fabricate the advanced non-noble-metal catalysts for NRR. It is believed that more effective and stable electrocatalysts would be developed for boosting the NRR in the future. 
Energy efficiency is another efficacious way to alleviate the energy crisis. In the field of energy-saving optoelectronics, electrochromic devices (ECDs) have shown great advantages. Among various fabrication materials for ECDs, coordination polymer (CP) shows a broad application prospect due to good cycle stability, high color rendering efficiency, and fast switching speed. Liu et al. present a comprehensive survey of the current achievements and progresses of CP in energy efficient ECDs from the aspect of influence of composition, coordination bonding and microstructure of pyridine-based $\mathrm{CP}$ on the performance of ECDs. This work is expected to provide the guideline for achieving a substantial enhancement in electrochromic and other optoelectronic fields.

Nevertheless, one of the paramount challenges to develop new high-efficiency energy transformation materials is the long span from experiment to practical application, due to the complexity of research objects and methods, insufficient personal accumulated experience, etc. (Luo et al.) Artificial intelligence (AI) has potential for solving the problems mentioned above. Luo et al. investigated and summarized research works on energy storage materials for capacitors and Li-ion batteries. They pointed out that machine learning (ML), as a subset of AI, algorithms can reduce test number of cycles and required experiments, which greatly reduces time consumption and accelerates every stage of development. In addition, they summarized the status and progress of AI in energy storage materials and present solutions to relevant deficiencies, such as the establishment of a database, extracting data from unstructured literature with automaticity and high efficiency and accuracy, etc. Apart from saving time, AI can predict the performance of materials, monitor reaction processes, and explore reaction mechanisms (Luo et al.; Yang et al.). Focusing on the superiority of AI in predicting experiments, Yang et al. reviewed the situation and application of $\mathrm{AI}$ in respects of optoelectronic materials, hydrogen peroxidation catalysts, water

\section{REFERENCES}

Centi, G. (2020). Smart catalytic materials for energy transition. SmartMat. 1:e1005. doi: 10.1002/smm2.1005

Chen,. C., Yan, X., Liu, S., Wu, Y., Wan, Q., Sun, X., et al. (2020). Highly efficient electroreduction of $\mathrm{CO}_{2}$ to $\mathrm{C}_{2}+$ alcohols on heterogeneous dual active sites. Angew. Chem. Int. Ed. 59, 16459-16464. doi: 10.1002/anie.202006847

Cheng, F., and Chen, J. (2012). Metal-air batteries: from oxygen reduction electrochemistry to cathode catalysts. Chem. Soc. Rev. 41, 2172-2192. doi: $10.1039 / \mathrm{clcs} 15228 \mathrm{a}$

Edwards, P. P., Kuznetsov, V. L., David, W. I. F., and Brandon, N. P. (2008). Hydrogen and fuel cells: towards a sustainable energy future. Energy Policy 36, 4356-4362. doi: 10.1016/j.enpol.2008.09.036

Hu, H., Wang, Z., Cao, L., Zeng, L., Zhang, C., Lin, W., et al. (2021). Metalorganic frameworks embedded in a liposome facilitate overall photocatalytic water splitting. Nat. Chem. 13, 358-366. doi: 10.1038/s41557-020-00635-5

Lee, J. C., Kim, J. Y., Joo, W. H., Hong, D., Oh, S. H., Kim, B., et al. (2020). Thermodynamically driven self-formation of copper-embedded nitrogen-doped carbon nanofiber catalysts for a cascade electroreduction of carbon dioxide to ethylene. J. Mater. Chem. A. 8, 11632-11641. doi: 10.1039/D0TA03322G

Li, Y., Li, J., Huang, J., Chen, J., Kong, Y., Yang, B., et al. (2021). Boosting electroreduction kinetics of nitrogen to ammonia via tuning electron electrolysis catalysts and microbial fuel cells. It indicates that the relationship between prediction and actual experiments is mutually facilitating. In other words, the efficiency of actual material processing can be promoted with accurate prediction, and the database for AI is extended.

In conclusion, the development of advanced materials and technologies for energy conversion and storage are of vital importance. Until now, various promising materials with excellent performance have been prepared, such as carbon nanomaterials [nanofibers (Zhao et al., 2018; Lee et al., 2020), nanotubes (Ma et al., 2019; Sun et al., 2020; Tuo et al., 2020; Zhang et al., 2020), graphene (Chen et al., 2020), etc.], reticular structure [metal-organic framework (Nam et al., 2018), covalent organic framework (Lin et al., 2015)], and tandem catalyst (Morales-Guio et al., 2018), etc. It is worth mentioning that traditional technologies in detection and characterization are gradually substituted with new and advanced solutions, such as computer science (AI, ML, etc.), and insitu characterization (In-situ/operando synchrotron radiation, in-situ/operando morphology/spectrum, etc.). It is believed the emerging technologies for materials design and characterization in energy conversion and storage will be greatly developed in the future.

\section{AUTHOR CONTRIBUTIONS}

ZZ supervised the project. JL wrote the manuscript. ZZ, YC, and $Z F$ revised the manuscript. All authors contributed to the article and approved the submitted version.

\section{FUNDING}

This work was supported by the National Natural Science Foundation of China (22071172). distribution of single-atomic iron sites. Angew. Chem. Int. Ed. 60, 9078-9085. doi: 10.1002/anie.202100526

Lin, S., Diercks, C. S., Zhang, Y., Kornienko, N., Nichols, E. M., Zhao, Y., et al. (2015). Covalent organic frameworks comprising cobalt porphyrins for catalytic $\mathrm{CO}_{2}$ reduction in water. Science 349, 1208-1213. doi: $10.1126 /$ science.aac8343

Liu, H., Zhu, Y., Ma, J., Zhang, Z., and Hu, W. (2020). Recent advances in atomic-level engineering of nanostructured catalysts for electrochemical $\mathrm{CO}_{2}$ reduction. Adv. Func. Mater. 30:1910534. doi: 10.1002/adfm.201910534

Ma, C., Hou, P., Wang,. X., Wang, Z., Li, W., and Kang, P. (2019). Carbon nanotubes with rich pyridinic nitrogen for gas phase $\mathrm{CO}_{2}$ electroreduction. Appl. Catal. B-Environ. 250, 347-354. doi: 10.1016/j.apcatb.2019.03.041

Ma, D., Han, S., Cao, C., Wei, W., Li, X., Chen, B., et al. (2021). Bifunctional singlemolecular heterojunction enables completely selective $\mathrm{CO}_{2}$-to-CO conversion integrated with oxidative 3D nano-polymerization. Energy Environ. Sci. 14, 1544-1552. doi: 10.1039/D0EE03731A

Morales-Guio, C. G., Cave, E. R., Nitopi, S. A., Feaster, J. T., Wang, L., Kuhl, K. P., et al. (2018). Improved $\mathrm{CO}_{2}$ reduction activity towards $\mathrm{C}_{2+}$ alcohols on a tandem gold on copper electrocatalys. Nat. Catal. 1, 764-771. doi: 10.1038/s41929-018-0 139-9

Nam, D., Bushuyen, O. S., Li, J., Luna, P. D., Seifitokaldani, A., Dinh, C. T., et al. (2018). Metal-organic frameworks mediate $\mathrm{Cu}$ coordination 
for selective $\mathrm{CO}_{2}$ electroreduction. J. Am. Chem. Soc. 140, 11378-12386. doi: 10.1021/jacs.8b06407

Ozdemir, J., Mosleh, I., Abolhassani, M., Greenlee, L. F., Beitle, R. R., and Beyzavi, M. H. (2019). Covalent organic frameworks for the capture, fixation, or reduction of $\mathrm{CO}_{2}$. Front. Energy Res. 7:77. doi: 10.3389/fenrg.2019.00077

Park, S., Shao, Y., Liu,. J., and Wang, Y. (2012). Oxygen electrocatalysts for water electrolyzers and reversible fuel cells: status and perspective. Energy Environ. Sci. 5, 9331-9344. doi: 10.1039/c2ee22554a

Sun, X., Zhang, Q., L, Q., Zhang, X., Shao, X., Yi, J., et al. (2020). Utilization of carbon nanotube and graphene in electrochemical $\mathrm{CO}_{2}$ reduction. Biointerface Res. Appl. Chem. 10, 5815-5827. doi: 10.33263/BRIAC104.815827

Tang, C., and Qiao, S. (2019). How to explore ambient electrocatalytic nitrogen reduction reliably and insightfully. Chem. Soc. Rev. 48, 3166-3180. doi: 10.1039/C9CS00280D

Tuo, J., Lin, Y., Zhu, Y., Jiang, H., Li, Y., Cheng, L., et al. (2020). Local structure tuning in $\mathrm{Fe}-\mathrm{N}-\mathrm{C}$ catalysts through support effect for boosting $\mathrm{CO}_{2}$ electroreduction. Appl. Catal. B-Environ. 272:118960. doi: 10.1016/j.apcatb.2020.118960

Wan, Y., Xu, J., and Lv, R. (2019). Heterogeneous electrocatalysts design for nitrogen reduction reaction under ambient conditions. Mater. Today 27, 69-90. doi: 10.1016/j.mattod.2019.03.002

Wang, N., Miao, R., Lee, G., Vomiero, A., Sinton, D., Ip, A. H., et al. (2021). Suppressing the liquid product crossover in electrochemical $\mathrm{CO}_{2}$ reduction. SmartMat. 2, 12-16. doi: 10.1002/smm2.1028

Wu, D., Kusada, K., Yoshioka, S., Yamamoto, T., Toriyama, T., Matsumura, S., et al. (2021). Efficient overall water splitting in acid with anisotropic metal nanosheets. Nat. Commun. 12:1145. doi: 10.1038/s41467-021-20956-4

Yang, C., Li, S., Zhang, Z., Wang, H., Liu, H., Jiao, F., et al. (2020a). Organicinorganic hybrid nanomaterials for electrocatalytic $\mathrm{CO}_{2}$ reduction. Small 16:2001847. doi: 10.1002/smll.202001847
Yang, C., Zhu, Y., Liu, J., Qin, Y., Wang, H., Liu, H., et al. (2020b). Defect engineering for electrochemical nitrogen reduction reaction to ammonia. Nano Energy 77:105126. doi: 10.1016/j.nanoen.2020.10 5126

Zhang, F., Hu, Y., Sun, R., Fu, H., and Peng, K. (2019). Goldsensitized silicon/ZnO core/shell nanowire array for solar water splitting. Front. Chem. 7:206. doi: 10.3389/fchem.2019.0 0206

Zhang, T., Han, X., Yang, H., Han, A., Hu, E., Li, Y., et al. (2020). Atomically dispersed Nickel(I) on an alloy-encapsulated nitrogen-doped carbon nanotube array for high-performance electrochemical $\mathrm{CO}_{2}$ reduction reaction. Angew. Chem. Int. Ed. 59, 12055-12061. doi: 10.1002/anie.202002984

Zhang, W., Low, J., Long, R., and Xiong, Y. (2019). Metal-free electrocatalysts for nitrogen reduction reaction. EnergyChem 2:100040. doi: 10.1016/j.enchem.2020.100040

Zhao, Y., Liang, J., Wang, C., Ma, J., and Wallace, G. G. (2018). Tunable and efficient Tin modified nitrogen-doped carbon nanofibers for electrochemical reduction of aqueous carbon dioxide. Adv. Energy. Mater. 8:1702524. doi: 10.1002/aenm.201702524

Conflict of Interest: The authors declare that the research was conducted in the absence of any commercial or financial relationships that could be construed as a potential conflict of interest.

Copyright (C) $2021 \mathrm{Li}$, Chen, Fan and Zhang. This is an open-access article distributed under the terms of the Creative Commons Attribution License (CC BY). The use, distribution or reproduction in other forums is permitted, provided the original author(s) and the copyright owner(s) are credited and that the original publication in this journal is cited, in accordance with accepted academic practice. No use, distribution or reproduction is permitted which does not comply with these terms. 